\title{
High-fidelity Characterization on Anisotropic Thermal Conductivity of Carbon Nanotube Sheets and on Their Effects of Thermal Enhancement of Nanocomposites
}

\author{
Xiao Zhang ${ }^{\mathrm{a}}(\bowtie)$, Wei Tan ${ }^{\mathrm{b}}$, Fiona Smail ${ }^{\mathrm{a}}$, Michael De Volder ${ }^{\mathrm{c}}$, Norman Fleck ${ }^{\mathrm{b}}$, Adam Boies ${ }^{\mathrm{a}}(\bowtie)$ \\ ${ }^{a}$ Division of Energy, Department of Engineering, University of Cambridge, Cambridge, CB2 1PZ, UK \\ ${ }^{b}$ Division of Mechanics, Department of Engineering, University of Cambridge, Cambridge, CB2 1PZ, UK \\ ${ }^{\mathrm{c}}$ Institute for Manufacturing, Department of Engineering, University of Cambridge, Cambridge, CB3 0FS, UK
}

\begin{abstract}
Some assemblies of nanomaterials, like carbon nanotube (CNT) sheet or film, always show outstanding and anisotropic thermal properties. However, there is still a lack of comprehensive thermal conductivity $(\kappa)$ characterizations on CNT sheets, as well as lack of estimations of their true contributions on thermal enhancement of polymer composites when used as additives. Always, these characterizations were hindered by the low heat capacity, anisotropic thermal properties or low electrical conductivity of assemblies and their nanocomposites. And the transient $\kappa$ measurement and calculations were also hampered by accurate determination of parameters, like specific heat capacity, density and cross-section, which could be difficult and controversial for nanomaterials, like CNT sheets. Here, to measure anisotropic $\kappa$ of CNT sheets directly with high fidelity, we modified the conventional steady-state method by measuring under vacuum and by infrared camera, and then comparing temperature profiles on both reference standard material and a CNT sheet sample. The highly anisotropic thermal conductivities of CNT sheets were characterized comprehensively, with $\kappa / \rho$ in alignment direction as $\sim 95$ $\mathrm{mW} \cdot \mathrm{m}^{2} /(\mathrm{K} \cdot \mathrm{kg})$. Furthermore, by comparing the measured thermal properties of different CNT-epoxy resin composites, the heat conduction pathway created by the CNT hierarchical network was demonstrated to remain intact after the in-situ polymerization and curing process. The reliable and direct $\kappa$ measurement rituals used here, dedicated to nanomaterials, will be also essential to assist in assemblies' application to heat dissipation and composite thermal enhancement.
\end{abstract}

\section{KEYWORDS}

Thermal conductivity, Carbon nanotube, Steady state, Composite, Nanomaterial, Heat dissipation 


\section{Introduction}

Due to the outstanding thermal properties of many nanomaterial, their corresponding assemblies, such as the carbon nanotube (CNT) sheet, fibres [11, 24], graphene and $\mathrm{BN}$ foams $[4,19]$, have been research hotspots for years in the field of heat dissipation and thermal conduction enhancement in polymer composites. However, reported experimental results have repeatedly shown the degradation of thermal properties when nanomaterials were used to form assemblies. For instance, thin films made from high quality CNTs show the in-plane thermal conductivity $(\kappa)$ to be $\sim 110 \mathrm{~W} /(\mathrm{K} \cdot \mathrm{m})$ and an improved $770 \mathrm{~W} /(\mathrm{K} \cdot \mathrm{m})$ for fibres after stretching and solvent densifying [7]. However, this $\kappa$ value is still much lower than that of an isolated SWCNT in both theoretical simulations $(6600 \mathrm{~W} /(\mathrm{K} \cdot \mathrm{m}))$ [2] and various experimental results $(2000-4000 \mathrm{~W} /(\mathrm{K} \cdot \mathrm{m})[6,13$, 26]) with different samples and measurement methods). The mechanisms that underlie the large discrepancy between bulk properties and individual CNTs have not been well described in literature, motivating detailed study of the processing and assembly of nanomaterial and their composite, to better take advantage of their outstanding thermal properties.

Among CNT assemblies, CNT sheet, with its outstanding performance, synthesized by use of the continuous floating catalyst chemical vapor deposition (FCCVD) method [14] has already become commercial with an industrial production?. With single layers of CNT aerogel emanating from tube furnace, collected on a roller, and finally densified by compression, a preferential alignment formed in plane of CNT sheets, as well as a multilayer structure out of plane. Consequently, CNT sheet shows highly anisotropic thermal and electrical properties.

However, to comprehensively study the anisotropic thermal properties in assemblies like CNT sheets, characterizations were always hindered by the lack of reliable measurement techniques, due to their incompetence on anisotropic thermal properties, as well as by low heat capacities and mass densities of assemblies.

For instance, with the frequently used laser flash technique [18], researchers deduced $\kappa$ from measured thermal diffusivity $(\alpha)$ with specific heat capacity $\left(c_{p}\right)$ and density $(\rho)$. However, the determination of an accurate $c_{p}$ and $\rho$ value is challenging for nanomaterials and their composites. This is reflected in the fact that scientific publications show a very large spread $(>20 \%)$ of $c_{p}$ values for CNTs [12], which is further complicated for $c_{p}$ of their resulting composites. Moreover, the transient measurement errors propagate when calculating $\kappa$, resulting in large uncertainties for nanomaterials. On the other hand, to measure $\kappa$ of nanomaterials directly, traditional steadystate methods also require modification. For example, inevitable thermal contact resistance has always led to erroneously lower $\kappa$ values. And nanomaterials' small heat capacities and thickness also leave temperature measurement susceptible to heat dissipation from temperature probes (e.g. thermocouples). Moreover, high $\kappa$ materials require longer samples to maintain a reliable temperature gradient, which increases the surface area for convective and radiative heat loss. These complications introduce non-steady effects, such as fluctuating heat loss from free convection, which must be avoided to achieve consistent results.

Furthermore, to evaluate the fillers or additives' thermal enhancement effect in a polymer composite, methods like the transient $3 \omega$ method or steady-state Joule-heating method [27], are not adaptable, due to complicated electrical resistance change of composites with temperature. Here we modified the conventional steady-state $\kappa$ measurement method, adapting it for nanomaterial measurement, and demonstrated the applicability of the technique by measuring CNT sheet and composites. We limit convective interference by conducting the measurements under vacuum. And all the temperature profiles were measured by an infrared camera to prevent heat dissipation and temperature disturbance from thermocouples. By taking both radiation and convection heat transfer into consideration, we measured $\kappa$ in plane (x and $y$ directions) with its confidence limit in various operating environments by stochastic error propagation calculation. With another steady state method, we calculated the $\kappa$ out of plane (z-direction) and the Biot number. Moreover, with the modified in plane $\kappa$ measurement technique, we measured thermal properties of different CNT-epoxy resin composites to study the CNT sheet filler's effect on thermal enhancement for polymer composites.

\section{Experimental Detail and Theoretical Basis for In Plane Measurement \\ 2.1 Experimental Detail}

As shown in Fig. 1 inset, to avoid the disturbance from free convection on nanomaterial samples, all the measurements were taken in a cylindrical aluminium vacuum chamber $(\varnothing$ $350 \mathrm{~mm} \times$ height $300 \mathrm{~mm}$ ) linked on the sidewall with a turbomolecule pump (PFEIFFER TPH 240PC). Optical access was achieved via a sidewall port, fitted with a $3 \mathrm{~mm}$ thick $\mathrm{ZnSe}$ optical window with anti-reflection layers on both sides. The window transmittance was calibrated to be approximately $92 \%$ at $7-14 \mu \mathrm{m}$.

A FLIR T650sc infrared camera $(640 \times 480$ LWIR resolution), equipped with 7.5-14 $\mu \mathrm{m}$ spectral band optics (f/1.0, focal length $24.6 \mathrm{~mm}$ ), as well as the ResearchIR software interface, was used to measure the temperature profile along samples. The noise-equivalent temperature difference (NETD) of $<20 \mathrm{mK}$ guaranteed high precision temperature resolution along the sample axis.

In Fig. 1, copper blocks were used as heat sinks at both hot and cold ends, whose temperatures were controlled by metal foil heaters (Kapton insulated) and K-type tinythermocouples (OMEGA SA3-K). The heat sink at the hot end (HotEnd) was thermally isolated by a $20 \mathrm{~mm}$ thick 


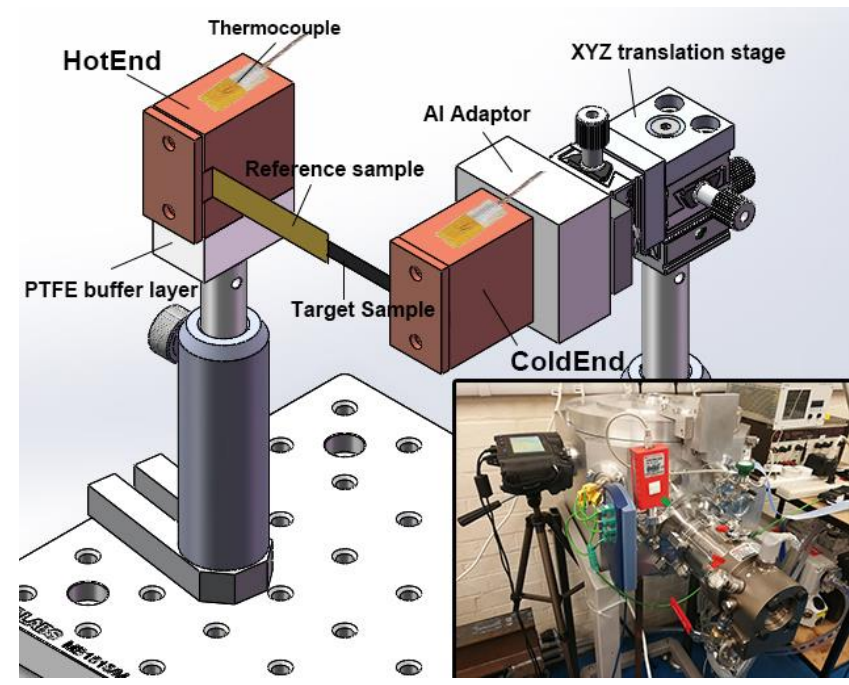

Figure 1 Schematic illustration of thermal conductivity measurement stage with copper hot and cold end, brass strip reference material and carbon nanotube sheet strip sample. Insert shows infrared camera measuring temperature through a sidewall port on the aluminium vacuum chamber $(\varnothing 350 \mathrm{~mm} \times$ height 300 $\mathrm{mm})$.

PTFE buffer layer. In contrast, the heat sink at the cold end (ColdEnd) was thermally linked to the vacuum chamber with an aluminium adaptor. The metallic reference material and nanomaterial sample (CNT sheet strip) were inserted into the HotEnd and ColdEnd, respectively. The other end of nanomaterial sample was attached onto the reverse side of the reference material with a thin layer of high thermal conductivity silver paint, forming the junction section (3$5 \mathrm{~mm}$ ). An XYZ translation stage at the ColdEnd eliminated the distortion on samples, to avoid disturbance of the thermal measurement.

For CNT sheet samples, we cut one large CNT sheet (thickness $\sim 70-100 \mu \mathrm{m}$ ) in different directions to obtain different anisotropic CNT sheet strip samples, with the length $\sim 50 \mathrm{~mm}$ in the $\mathrm{x}(\mathrm{y})$-direction, and the width of $5 \mathrm{~mm}$ in the $\mathrm{y}(\mathrm{x})$-direction.

By properly selecting a reference material with suitable thermal conductivity and geometry, comparable temperature gradients were achieved on both the reference material and the sample. For metallic materials, the emissivity was modified with a thin layer of black paint to guarantee a reliable temperature reading.

The measurement procedure began when all components reached a stable temperature (with temperature change no more than $0.1 \mathrm{~K}$ over $10 \mathrm{~min}$ ) under vacuum. Then, both the temperature profile along the reference material and nanomaterial were recorded by infrared camera with the mean temperature taken along the sample width (y direction). Temperatures of the ColdEnd, HotEnd, chamber wall and the atmosphere were simultaneously recorded by tiny thermocouples.

\subsection{Theory Basis}

Based on the low Biot number $(B i \ll 1)$ calculated in section 4 , we used the one-dimensional heat conduction equation (Eq. 1), accounting for convection and radiation heat loss, to model the temperature profile of the sample,

$$
\kappa A \frac{d^{2} T}{d x^{2}}-h_{c} P\left(T-T_{g}\right)-\varepsilon \sigma P\left(T^{4}-T_{\infty}{ }^{4}\right)=0
$$

$\kappa$ represents the thermal conductivity along the temperature gradient (x direction), $A$ represents the sample's cross-section area orthogonal to the heat transfer (y-z plane), $h_{c}$ is the convective heat transfer coefficient, $P$ is the sample's surface isothermal perimeter from which the convection (radiation) heat was transferred to the surrounding gas (environment), $\varepsilon$ was the sample's emissivity, $\sigma$ was the Stefan-Boltzmann constant, and $T$ represents the temperatures of the system, gas $\left(T_{g}\right)$ and environment $\left(T_{\infty}\right)$.

In previous reports, to solve Eq. 1, temperature at the two ends have been frequently used as the boundary conditions [25]. However, this choice could make $\kappa$ sensitive to temperature errors. Here, as shown in Fig. 2a, we followed the reported dual-mode method [15] to use the heat flux $Q_{i n}$ at the HotEnd as the first boundary condition (Eq. 2), which is proportional to the temperature gradient. The second boundary condition was the temperature at the ColdEnd (Eq. 3):

$$
\begin{aligned}
& -\left.\kappa A \frac{d T}{d x}\right|_{x=0}=Q_{i n} \\
& T(x=L)=T_{C E}
\end{aligned}
$$

where $L$ represents the length of the sample. This method makes the fitting process much more robust against parameter errors.

By maintaining the temperature ranges of sample and reference material's cold tail (in Fig. 2b, plot with orange background) within $10 \mathrm{~K}$ ranges, it was reasonable to consider $\kappa, h_{c}, \varepsilon$ as fixed values. Moreover, to simplify calculation, the radiation term was replaced by its 1 st order Taylor polynomial*:

$$
T^{4}-T_{\infty}{ }^{4} \approx 4 T_{\text {mean }}{ }^{3}\left(T-T_{\infty}\right)
$$

Above $T_{\text {mean }}$ was the mean temperature of target sample with surrounding shield. Within $10 \mathrm{~K}$ above room temperature $(\sim 297 \mathrm{~K})$, it is reasonable to treat $T_{\text {mean }}$ as a constant when solving the Eq. 1.

The solution for Eq. 1 is as follows:

$$
T=\frac{\left(T_{C E}-\frac{\beta}{\alpha}\right) \cosh (\omega x)+\frac{Q_{i n}}{\omega \kappa A} \sinh (\omega(L-x))}{\cosh (\omega L)}+\frac{\beta}{\alpha}
$$

where $\alpha=h_{c} P+H_{r} \varepsilon P, \quad \beta=h_{c} P T_{g}+H_{r} \varepsilon P T_{\infty}, \quad \omega=$ $\sqrt{\frac{\alpha}{\kappa A}}, H_{r}=4 T_{\text {mean }}^{3} \sigma$

Based on Eq. 5, $\kappa$ could be calculated by fitting the experimental temperature profile of the sample with all experimental parameters.

Considering energy conservation at the junction section, $Q_{\text {in }}$ could be determined by deducting radiation $\left(Q_{\text {rad }}\right)$ and 


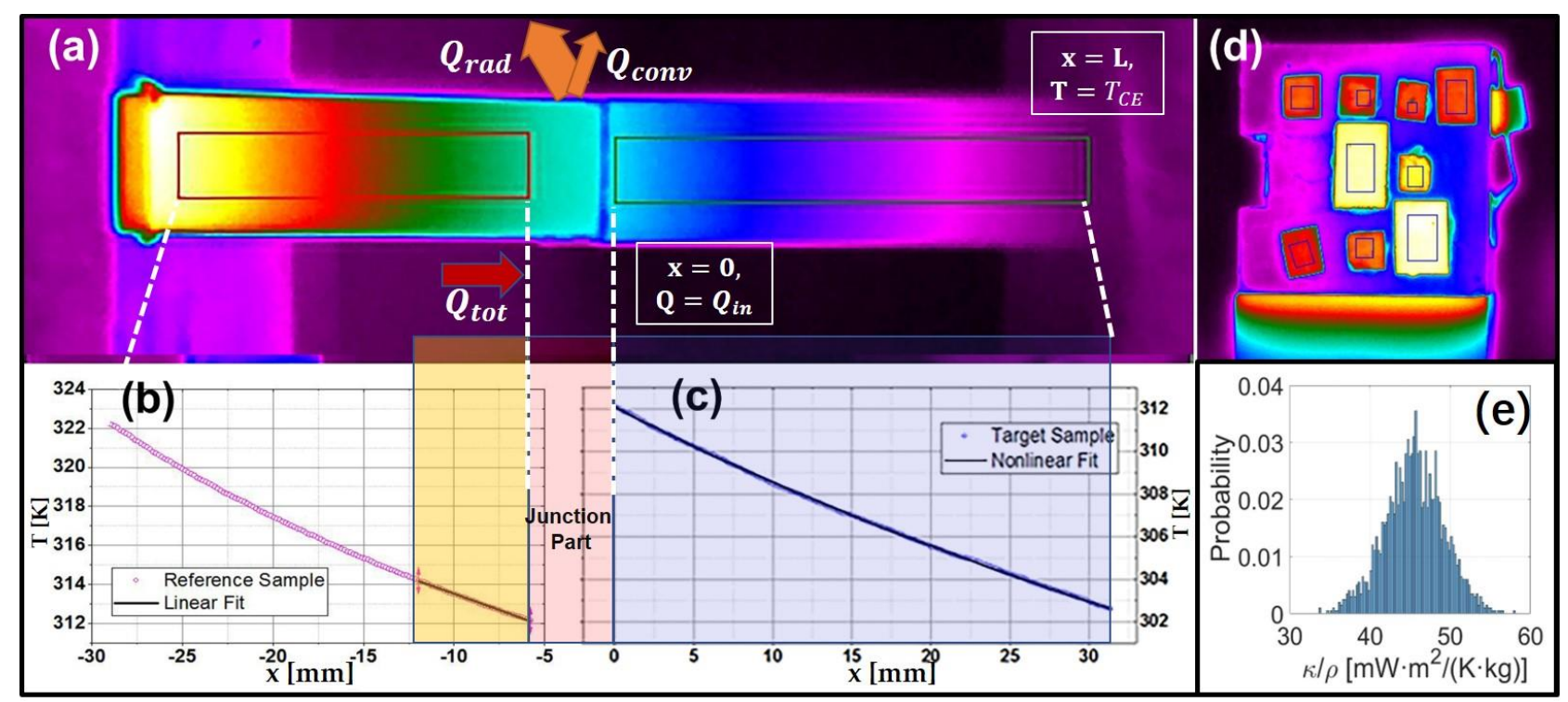

Figure 2 (a) Infrared temperature contour image during experiment, brass and copper strips were used as reference and target sample respectively, with a junction of approximately $5 \mathrm{~mm}$ between them; (b) Heat flow $\left(Q_{t o t}\right)$ into junction was calculated by linear fitting, based on the low temperature tail of the reference sample (plot with orange background); (c) thermal conductivity $\kappa$ was deduced by fitting of the temperature profile along the target sample; (d) the emissivities of various samples were adjusted to get accurate temperature reading; (e) with Stochastic error propagation modelling, $\kappa / \rho$ of copper was deduced to be $45 \pm 4 \mathrm{~mW} \cdot \mathrm{m}^{2} /(\mathrm{K} \cdot \mathrm{kg})$.

convection heat loss $\left(Q_{c o n v}\right)$ from the heat flow $Q_{t o t}$ into the junction section (out of the reference sample).

$$
Q_{\text {in }}=Q_{\text {tot }}-Q_{\text {conv }}-Q_{\text {rad }}
$$

\subsubsection{Stochastic error propagation calculation and sensitivity analysis}

During the fitting process, all geometric, thermal, and optical parameters had inherent uncertainties, including experimental random errors and systematic errors. All these errors led to the deviation of calculated $\kappa$ from the true $\kappa$ value.

Considering the practical engineering application demand, referential errors were also calculated by the stochastic error propagation method, generating confidence limits for each calculated $\kappa$ value, as shown in Fig. 2e. Briefly, during every round of iteration, $\kappa$ was fitted with a new set of random parameters which were generated based on parameters' expected values, errors, bounds and distribution style.

Meanwhile, by using Spearman's rank correlation coefficient, the correlation analysis between $\kappa$ and parameters' uncertainties was also conducted. Thus, the thickness and $\kappa$ of the reference sample, as well as $h_{c}$ were determined to reduce systematic errors during experiments.

\subsubsection{Thermal conductivity normalized by volume density}

For porous nanomaterials like the CNT sheet, it is challenging to obtain true thickness or solid volume. Incorrect thickness or volume is one of the major sources of reported $\kappa$ values variation.
To obtain more accurate results, we adopted thermal conductivity normalized by solid volume density $(\kappa / \rho)$, as the primary dimensions of reported results $\left[\mathrm{W} \cdot \mathrm{m}^{2} /(\mathrm{K} \cdot \mathrm{kg})\right]$. $\kappa / \rho$ was deduced from the measured value $\kappa \mathrm{A}$ in Eq. 5 as follows:

$$
\frac{\kappa}{\rho}=\kappa \div \frac{m}{l \cdot A l} \approx \kappa A \div \frac{m}{l}
$$

Here, $m / l$ was the mass per meter, and $A^{\prime}$ was the solid cross-sectional area in CNT sheet excluding all voids.

To compare our results with other literature values, thermal conductivity $\kappa$ could be deduced by incorporating a sample solid density. Regarding the CNT sheet, the true solid density of porous sheet $\rho$ is around $\sim 1.5 \mathrm{~g} / \mathrm{cm}^{3}[1]$.

As can be seen from Eq. 5 and $7, \kappa A$ is the value we measured by experiments and calculated without assumption of any material parameter. Here we make a reliable assumption: $A^{\prime} \approx A$, i.e., the cross-sectional area used in the density calculation is also the area through which heat is conducted. By this method, only reliable values conforming to the experiment have been used in the calculation of $\kappa$. The measured $\kappa$ value describes the heat conduction solely in the solid CNT accounting for the nanomaterials' porous structure.

\subsection{Calibration of emissivity and transparency}

All samples tested did not exhibit pure blackbody emissivities $(\varepsilon<1)$. By using an infrared camera, the accuracy of the detected temperature primarily relies on the accurate measurement of a samples' emissivity, as well as the window's transmittance at the measured temperature. $\varepsilon$ of all samples have been determined by following the 
procedures shown in ASTM E1933-14. Briefly, we attached various samples onto a large copper heat sink with highly thermal conductivity silver paint. The temperature of the heat sink was more than $20 \mathrm{~K}$ higher than that of the surrounding environment. The emissivity could be determined when the detected temperature of sample equaled that of the heat sink upon adjustment of $\varepsilon$. As can be seen from Fig. 2d, due to material difference and surface roughness, the colors in the infrared image were different. The calibrated $\varepsilon$ of CNT samples was approximately $0.7 \pm 0.1$.

With $\varepsilon$ of the samples and temperature of the chamber, the transmittance of window was determined by following procedures shown in ASTM E1897-14, and was found to be 0.92 at $7-14 \mu \mathrm{m}$ at room temperature.

\subsection{Calibration of convection heat transfer coefficient}

In high vacuum environments, heat dissipation mainly comes from radiation which can be evaluated from the Stefan-Boltzmann equation. To improve $\kappa$ measurement precision and compare the heat loss constituents from radiation and convection, we also measured the convective heat transfer coefficient (still represented by $h_{c}$ ) under 3 different gas pressures ( $6 \mu$ bar with turbomolecule pump, 0.2 mbar with only mechanical pump and atmospheric pressure), as shown in Table 1 . With $h_{c}$ calibrated on the standard material with known thermal conductivity under different pressures, $\kappa$ of the standard sample and nanomaterials were then calculated.

It is common knowledge that $h_{c}$ varies with gas density, viscosity, thermal conductivity, specific heat, and flow conditions [3]. To calibrate $h_{c}$, a brass strip $(0.025 \mathrm{~mm}$ 13505 Brass foil, Alloy 260 AlfaAesar) was mounted directly between the HotEnd and ColdEnd. The temperature profile along the whole brass strip was measured. The low temperature region (within $10 \mathrm{~K}$ ) was chosen as the "sample" section, and a short adjacent region with higher temperature was used as the "reference material" to calculate $Q_{\text {in }}$. There was no junction section between the "reference material" and the "sample" sections. By repeatedly pumping and venting with different gases, and based on the reported $\kappa$ of brass alloy 260 of $110 \mathrm{~W} /(\mathrm{K} \cdot \mathrm{m})$ [3], the $h_{c}$ of air, He and Ar under different pressures have been calibrated as shown in Table 1 .

Under atmospheric pressure, a thermal boundary layer will develop in gas adjacent to the vertical heated sample. Based on the calculated Rayleigh number ( $R a$, Method A-1 in the ESM), the $R a$ was around 30-400, which was far below the critical $R a=10^{9}$ to develop a turbulent boundary layer. Thus, a stable laminate boundary layer will develop along samples. Based on empirical functions [3, 5], average Nusselt number $(\overline{N u})$ and average convective coefficient $\left(\overline{h_{c}}\right)$ could be deduced (Method A-1 in the ESM). Besides, if treated as a constant, $h_{c}$ at 1 bar was calibrated to be $\sim 8.6 \mathrm{~W} /\left(\mathrm{K} \cdot \mathrm{m}^{2}\right)$ for air, close to the value calculated by empirical correlations, as shown in Table 1 . In contrast, the
Table 1 Convection heat transfer coefficient calibrated by standard brass material

\begin{tabular}{c|c|c|c|c} 
Gas & $\begin{array}{c}h_{c} \\
\text { at } 6 \mu \mathrm{bar} \\
{\left[\mathrm{W} /\left(\mathrm{K} \cdot \mathrm{m}^{2}\right)\right]}\end{array}$ & $\begin{array}{c}h_{c} \\
\text { at } 0.2 \mathrm{mbar} \\
{\left[\mathrm{W} /\left(\mathrm{K} \cdot \mathrm{m}^{2}\right)\right]}\end{array}$ & $\begin{array}{c}h_{c} \\
\text { at } 1 \mathrm{bar}^{\mathrm{a}} \\
{\left[\mathrm{W} /\left(\mathrm{K} \cdot \mathrm{m}^{2}\right)\right]}\end{array}$ & $\begin{array}{c}\text { Empirical } \\
h_{c} \text { at } 1 \text { bar } \\
{\left[\mathrm{W} /\left(\mathrm{K} \cdot \mathrm{m}^{2}\right)\right]}\end{array}$ \\
\hline Air & $0.068 \pm 0.140$ & $0.75 \pm 0.55$ & $8.6 \pm 2.2$ & $7.1-10.8$ \\
\hline $\mathrm{He}$ & $0.059 \pm 0.131$ & $1.07 \pm 0.63$ & $16.4 \pm 2.9$ & $9.1-16.9$ \\
\hline $\mathrm{Ar}$ & $0.057 \pm 0.141$ & $0.50 \pm 0.46$ & $4.5 \pm 1.4$ & $2.9-5.8$ \\
\hline
\end{tabular}

a The free convection coefficient $h_{c}$ at 1 bar should change with sample's width and surface temperature. Herein, all data was calibrated based on a brass strip with $7 \mathrm{~mm}$ in width within $10 \mathrm{~K}$ above room temperature ( $297 \mathrm{~K})$

higher $h_{c}$ of helium gas resulted in the target sample decreasing to the gas temperature over a shorter length range. This was a consequence of the different gas densities at 1 bar and a much different $\kappa$ at $300 \mathrm{~K}$ (Table S1. in the ESM).

Under $6 \mu \mathrm{bar}, h_{c}$ of argon and helium showed no obvious difference to air. This may be the result of the molecular ballistic motion becoming increasingly dominant under vacuum. Under $0.2 \mathrm{mbar}, h_{c}$ of air was $0.75 \pm 0.55$ $\mathrm{W} /\left(\mathrm{K} \cdot \mathrm{m}^{2}\right)$, which was similar to the reported $0.8 \mathrm{~W} /\left(\mathrm{K} \cdot \mathrm{m}^{2}\right)$ at 0.18 mbar [21]. The mean free path $\left(\lambda_{m f p}\right)$ of air molecules is $\sim 0.34 \mathrm{~mm}$, which is large enough to avoid air molecules being constrained in micro/nanoscale surface geometry [3]. Similar $h_{c}$ relationship changing with gases with that under atmospheric pressure, manifests the transition from viscous flow to molecule flow.

Under atmospheric pressure, the mean free path $\left(\lambda_{m f p}\right)$ for air, is $\sim 66 \mathrm{~nm}$. Considering the influence on $h_{c}$ by various surface geometry of samples, as well as multiple choices of empirical correlations, although $\kappa$ could also be roughly measured under atmospheric pressure with empirical $h_{c}$, most of our experiments have been finished under vacuum condition around $6 \mu \mathrm{bar}$, under which $\lambda_{m f p} \sim 11 \mathrm{~mm}$. For nanomaterials with high emissivity (like CNT sheets with $\varepsilon \sim 0.7)$, the typical radiation heat loss coefficient $\left(H_{r} \varepsilon\right)$ was approximately $5 \pm 1 \mathrm{~W} /\left(\mathrm{K} \cdot \mathrm{m}^{2}\right)$. Since $h_{c}$ is less than $3 \%$ of $H_{r} \varepsilon$, the major heat dissipation route under $6 \mu$ bar was indeed radiation. Thanks to less heat loss from convection, temperature profile could be measured more precisely. Moreover, much smaller $h_{c}$ under vacuum giving a smaller $B i$ number, also guarantees a more uniform temperature distribution on sample cross-section (section 4).

\section{Results and Discussion for In Plane Measurement 3.1 Setup verification using standard materials}

To verify the feasibility of our setup, as shown in Fig. 2a, a brass strip $(0.13 \pm 0.01 \mathrm{~mm}$ thick, 13504 Alloy 260 Brass foil, AlfaAesar) and a copper strip $(0.025 \pm 0.003 \mathrm{~mm}$ thick, 46986 99.8\% Copper foil, AlfaAesar) were used as the reference material and the sample, respectively.

Based on reported $\kappa=110 \mathrm{~W} /(\mathrm{K} \cdot \mathrm{m})$ of brass alloy 260 [3], the $\kappa / \rho$ of copper was measured under vacuum as $45.5 \pm 3.7 \mathrm{~mW} \cdot \mathrm{m}^{2} /(\mathrm{K} \cdot \mathrm{kg})$, i.e. $\quad \kappa=407.5 \pm 33.7 \mathrm{~W} /(\mathrm{K} \cdot \mathrm{m})$ 
(with density of $8.96 \mathrm{~g} / \mathrm{cm}^{3}$ ), compared to the reported 401 $\mathrm{W} /(\mathrm{K} \cdot \mathrm{m})$ [3]. The main error came from the error of standard material's thickness $(0.025 \pm 0.003 \mathrm{~mm})$. However, when measured at atmospheric pressure, and using the empirical $h_{c}$ of free convection, the $\kappa / \rho$ of copper was measured as $43.6 \pm 4.8 \mathrm{~mW} \cdot \mathrm{m}^{2} /(\mathrm{K} \cdot \mathrm{kg})$, i.e. $\kappa=390.5 \pm 43.0$ $\mathrm{W} /(\mathrm{K} \cdot \mathrm{m})$. Due to the reasons mentioned in the end of section 2.4, the following measurements were all conducted under $6 \mu$ bar vacuum condition to obtain more consistent results.

\subsection{In Plane Thermal Conductivity of Thick CNT Sheets} All of the CNT sheet samples tested in our system were synthesized by use of the continuous FCCVD method [14], supplied by Tortech Nanofibers Ltd ${ }^{\top}$. Single layers of CNT aerogel emanating from tube furnace were collected on a roller, and finally densified by compression. Due to the gas flow drag force during the synthesis process and a small tension force applied during the collecting process, CNT bundles formed a preferential alignment direction in CNT sheets. Here the directions parallel with and perpendicular to the alignment direction in the CNT sheet are called the $\mathrm{x}$-direction and $\mathrm{y}$-direction, respectively. By cutting in different directions from one large CNT sheet, we obtained CNT strip samples with the length in the $\mathrm{x}(\mathrm{y})$-direction, and the width in the $\mathrm{y}(\mathrm{x})$-direction. The sample was linked with $0.025 \mathrm{~mm}$ thick reference brass foil strips with junction section approximately $5 \mathrm{~mm}$ in length direction. Based on the above steady-state measurement routine, the results showed that, in the $\mathrm{x}$-direction, the as-received CNT sheet reached $\kappa / \rho=94.9 \pm 20.1 \mathrm{~mW} \cdot \mathrm{m}^{2} /(\mathrm{K} \cdot \mathrm{kg})$ due to simple densification via compression. In the $y$-direction, $\kappa /$ $\rho=84.4 \pm 17.5 \mathrm{~mW} \cdot \mathrm{m}^{2} /(\mathrm{K} \cdot \mathrm{kg})$. The lower value in the $\mathrm{y}-$ direction was due to fewer CNTs bundles in the heat conduction pathway compared to the $\mathrm{x}$-direction. Compared to the result in section 3.1 , the $\kappa / \rho$ of CNT sheet was approximately 2 times of that of pure copper. This high value also confirmed our motivation to develop a steady-state method for highly thermal conductivity materials.

Based on the solid density of SWCNT $1.5 \mathrm{~g} / \mathrm{cm}^{3}$ [1], $\kappa$ could be deduced to be $142 \pm 30 \mathrm{~W} /(\mathrm{K} \cdot \mathrm{m})$ in the $\mathrm{x}$-direction and $\kappa=127 \pm 26 \mathrm{~W} /(\mathrm{K} \cdot \mathrm{m})$ in the $\mathrm{y}$-direction, both of which are still much lower than the value of isolated SWCNT $(\kappa=\sim 3000 \mathrm{~W} /(\mathrm{K} \cdot \mathrm{m})[25])$. The difference originates mainly from the disorientation of CNT in the sheet (as shown in Fig. S-1 in the Electronic Supplementary Material (ESM)) and the phonon quenching at boundaries, between impurities, as well as defects etc. By using the material density of solid CNT, the above conductivity reported here purposely omits the air voids within the CNT material both within an individual CNT and between CNTs.

The CNT sheet can be regarded as a composite of CNT and air inside. In the experiment, $\kappa A$ was measured. The effective thermal conductivity $\kappa_{C N T}^{e f f}$ could be calculated by estimating a rectangular cross-sectional area, that is, to treat the sheet as a homogenous material. The relationship between $\kappa_{C N T}^{e f f}$ and the true thermal conductivity of CNT network could be roughly described as follows [9],

$$
\kappa_{C N T}^{e f f}=\phi \kappa_{C N T}+(1-\phi) \kappa_{\text {air }}
$$

Although the volume fraction $\phi$ of air in the CNT sheet was higher than that of CNT (as shown in Table 2), since $\kappa_{\text {air }}$ is too low in comparison to $\kappa_{C N T}$, the contribution of air in the thermal dissipation is negligible. Here by using the calculation method shown in section 2.2.2, all the $\kappa$ results reported here were $\kappa_{C N T}$ instead of $\kappa_{C N T}^{e f f}$.

Table 2. Thermal conductivity in x-direction of composites with different epoxy content.

\begin{tabular}{|c|c|c|c|c|c|c|c|c|c|c|}
\hline Samples & $\begin{array}{l}t_{C N T} \\
{[\mu \mathrm{m}]}\end{array}$ & $\begin{array}{c}t_{e f f} \\
{[\mu \mathrm{m}]}\end{array}$ & $\begin{array}{c}\rho_{\text {eff }} \\
{\left[\mathrm{g} / \mathrm{cm}^{3}\right]}\end{array}$ & $\begin{array}{l}\text { CNT } \\
\text { wt } \%\end{array}$ & $\begin{array}{l}\text { CNT } \\
\text { vol\% }\end{array}$ & $\begin{array}{c}\text { Epoxy } \\
\text { vol\% }\end{array}$ & $\begin{array}{c}\text { Air } \\
\text { vol\% }\end{array}$ & $\begin{array}{c}\kappa A / w / t_{C N T} \\
{[\mathrm{~W} /(\mathrm{K} \cdot \mathrm{m})]}\end{array}$ & $\begin{array}{c}\kappa_{c p}^{e f f^{a}} \\
{[\mathrm{~W} /(\mathrm{K} \cdot \mathrm{m})]}\end{array}$ & $\begin{array}{c}\kappa_{C N T}^{b} \\
{[\mathrm{~W} /(\mathrm{K} \cdot \mathrm{m})]}\end{array}$ \\
\hline Pure CNT & 111 & 113 & 0.33 & $100 \%$ & $19.5 \%$ & $0 \%$ & $80.5 \%$ & $33.1 \pm 6.4$ & $32.6 \pm 6.3$ & $167 \pm 32$ \\
\hline Composite \#1 & 107 & 89 & 0.44 & $98.9 \%$ & $25.2 \%$ & $0.4 \%$ & $74.4 \%$ & $32.4 \pm 6.1$ & $39.0 \pm 7.4$ & $155 \pm 29$ \\
\hline Composite \#2 & 106 & 86 & 0.47 & $92.8 \%$ & $25.5 \%$ & $3.0 \%$ & $71.5 \%$ & $33.5 \pm 6.1$ & $41.4 \pm 7.6$ & $162 \pm 30$ \\
\hline Composite \#3 & 96 & 73 & 0.72 & $65.2 \%$ & $25.6 \%$ & $21.0 \%$ & $53.4 \%$ & $34.6 \pm 6.6$ & $45.2 \pm 8.6$ & $176 \pm 33$ \\
\hline Composite \#4 & 98 & 62 & 1.21 & $44.0 \%$ & $30.3 \%$ & $59.1 \%$ & $10.6 \%$ & $32.9 \pm 6.8$ & $51.6 \pm 10.7$ & $170 \pm 35$ \\
\hline Composite \#5 & 124 & 129 & 1.23 & $21.4 \%$ & $14.7 \%$ & $83.2 \%$ & $2.1 \%$ & $29.6 \pm 5.7$ & $28.4 \pm 5.5$ & $191 \pm 35$ \\
\hline Composite \#6 & 131 & 149 & 1.19 & $19.8 \%$ & $13.7 \%$ & $85.5 \%$ & $0.8 \%$ & $26.6 \pm 5.1$ & $23.5 \pm 4.5$ & $169 \pm 31$ \\
\hline
\end{tabular}

${ }^{a} \kappa_{c p}^{e f f}$ of composites were calculated by dividing experimental results $\kappa A$ with sample strips' width $\mathrm{w}$ and $t_{e f f}$ of composites measured by a micrometer;

${ }^{\mathrm{b}} \kappa_{\mathrm{CNT}}$ is calculated by solving $\kappa_{c p}^{e f f}=\phi_{C N T} \kappa_{C N T}+\phi_{\text {epoxy }} \kappa_{\text {epoxy }}+\phi_{\text {air }} \kappa_{\text {air }}$, here $\phi$ is the volume fraction and $\kappa_{\text {epoxy }}=0.35 \mathrm{~W} /(\mathrm{K} \cdot \mathrm{m}), \kappa_{\text {air }}=0.026$

$\mathrm{W} /(\mathrm{K} \cdot \mathrm{m})$ For pure CNT, $\kappa_{C N T}$ could also be calculated by Eq.7. which was $153 \pm 31 \mathrm{~W} /(\mathrm{K} \cdot \mathrm{m})$. 


\subsection{Thermal Conductivity of CNT thick sheet reinforced polymer composite}

The CNT sheet reinforced composite was fabricated by submerging CNT sheets into epoxy/acetone mixtures (varying the epoxy concentration) for 1 minute to make a CNT sheets prepreg. These resin-impregnated CNT sheets were then cured for $3 \mathrm{~h}$ at $120^{\circ} \mathrm{C}$ in an oven under pressure of 6 bar, followed by oven cooling to room temperature (Figure S-2 in the ESM).

Here with the same thermal conductivity measurement technique, we measured the $\kappa A$ of cured CNT sheet/epoxy composite with different epoxy content. By using a brass foil reference material, the apparent temperature gradient could be recorded along the composite length.

As heterogeneous materials, composites' density increase roughly with the increasing of epoxy content. Consequently, the routine of deducing $\kappa$ from $\kappa / \rho$ mentioned in section 2.2.2 may also include additional errors. To illustrate the influence of epoxy on the heat conduction ability of the composite, we can compare the value of $\kappa A / w / t_{C N T}$, that is $\kappa A$ normalized by CNT strips' width $(\mathrm{w})$ and thickness of CNT sheet $\left(t_{C N T}\right.$, thickness of CNT sheet measured by micrometer before submerged by epoxy).

As shown in Table 2 and Fig S- $3 \mathrm{a}, \kappa A / w / t_{C N T}$ of the composite remained nearly unchanged from adding small amount of epoxy resin on CNT sheet to totally submerging CNT in epoxy. Considering epoxy's low thermal conductivity, the preservation of $\kappa A / w / t_{C N T}$ from pure CNTs to composites indicated that polymer encapsulation of the CNTs has not degraded heat conduction of the composites. Increased phonon scattering at the polymer matrix and CNTs [17] were not detectable here. As shown in Table 2, the $\kappa_{C N T}$ deduced from thermal conductivity of composites $\kappa_{c p}^{e f f}$ also indicates a similar trend with $\kappa A / w / t_{C N T}$. These results indicate that the heat conduction pathway in the composite had not been degraded after the in-situ polymerization and curing process [20].

Furthermore, $\kappa_{c p}^{e f f}$ of composites increased monotonously with volume fraction of CNT sheet increase in composites (Fig S-3b and Table 2.). With maximum CNT volume fraction as $\sim 30 \%$, the thermal conductivity reached a much high value relative to typical CNT/epoxy composites, $\kappa_{c p}^{e f f}=51.6 \pm 10.7 \mathrm{~W} /(\mathrm{K} \cdot \mathrm{m})$ as compared to $0.24-5.5 \mathrm{~W} /(\mathrm{K} \cdot \mathrm{m})$ [10]. The high thermal conductivities of the CNT sheet/epoxy composite result from the interconnected CNT bundles hierarchical network produced from FCCVD which offers a continuous heat conduction pathway (Figure S-1). In the sheet, the interface between CNTs is longitudinal, providing 1D linear contact, which reduces the thermal interface resistance between CNTs when compared to the point contact found in short CNTs reinforced composites

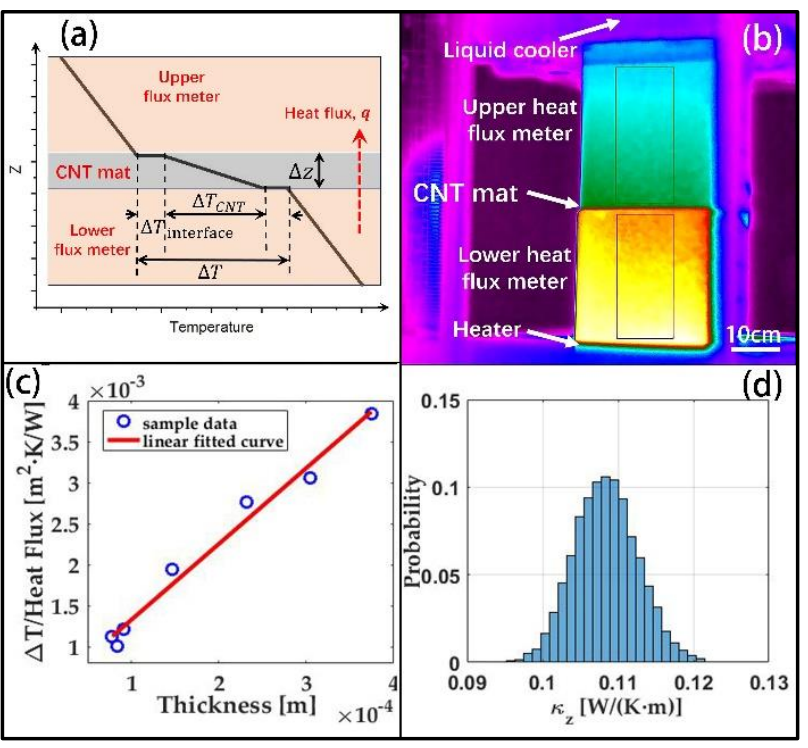

Figure 3 (a) Schematic depicting the temperature profiles of Bisubstrate technique [22]; (b) Infrared temperature contour image during measurement by using the modified steady-state Bisubstrate technique to measure the z-direction $\kappa$ of pure CNT sheets; (c, d) Linear fitting and stochastic error propagation calculation of data from 3 samples with different thickness, $\kappa_{z}$ was measured as $108 \pm 4 \mathrm{~mW} / \mathrm{m} / \mathrm{K}$.

[10]. The geometry of the CNT sheet composite is similar to that of the synergistic thermal enhancement effect of the CNTs and graphene nanoplates [23]. By contrast, in CNTs powder reinforced composites, CNTs transported heat through overcoming CNT-Polymer thermal interface resistance [8]. Additionally, by compressing the CNT aerogel and in-situ polymerization, the CNT volume fraction in our composites $(>20 \%)$ is much higher than that of CNT powder reinforced composites $[10,16]$, and therefore delivers high thermal conductivity per unit volume.

\section{Out of Plane Thermal Conductivity Measurement and Biot Number Calculation}

As mentioned in section 3.2, to synthesize the CNT sheet by using the continuous FCCVD method, the CNT aerogel was collected layer upon layer onto a roller, and finally densified by compression. Consequently, in the out of plane direction (z-direction) of the CNT sheet, there are many interfaces between CNT aerogel layers. Hence, $\kappa$ in the z-direction was envisaged much lower than that of the $\mathrm{x} / \mathrm{y}$-direction.

As a result, it was necessary to confirm the assumptions of theory mentioned in section 2.2, i.e. one-dimensional heat conduction and uniform temperature distribution within nanomaterials assembly. Here we calculated the $B i$ number, which provides a criterion of the temperature drop in the solid with depth, relative to the temperature difference between the solid's surface and the environment, under convective and radiative transfer [3]. 
In particular, if $B i \ll 0.1$, the resistance to conduction within the solid is much less than the resistance to heat loss to the surroundings.

According to Fig. 2(a), Bi along the width and z-direction should have forms as follows [3]:

$$
\begin{gathered}
B i_{w}=w(w+t)\left(h_{c}+H_{r} \varepsilon\right) /\left(2 t \kappa_{y}\right) \\
B i_{z}=t(w+t)\left(h_{c}+H_{r} \varepsilon\right) /\left(2 w \kappa_{z}\right)
\end{gathered}
$$

Above, $w$ and $t$ are the width and thickness of the sample, $\kappa_{w}$ and $\kappa_{z}$ were the thermal conductivities in the width-direction and $z$-direction. In section 3.2, we have already measured thermal conductivity in the $\mathrm{x} / \mathrm{y}-$ direction. To measure the low thermal conductivity in the z-direction, we use another steady-state technique, the Bisubstrate technique [22]. Briefly, as shown in Fig. 3(a), 2 substrates were used as heat flux meters, $\kappa_{z}$ and interface thermal resistance could be calculated by linear fitting data obtained from samples with different thickness by using the following equation:

$$
\frac{\Delta T}{q}=\frac{\Delta z}{\kappa_{z}}+\frac{2}{R_{i}}
$$

where $\Delta T$ was the total temperature drop, $q$ was heat flux through sample, $\Delta z$ was samples' thickness, and $R_{i}$ was the interface thermal resistance.

As shown in Fig. 3(b), temperature profiles along copper heat flux meters above and beneath thin CNTs samples were recorded by infrared camera. In Fig. 3(c, d), by linear fitting data from CNT sheets with thicknesses of 78, $85,92,147,232,305$ and $374 \mu \mathrm{m}$, and stochastic error propagation modelling, we measured $\kappa_{z}$ of CNT sheets as $108 \pm 4 \quad \mathrm{~mW} /(\mathrm{K} \cdot \mathrm{m})$ with $R_{i} \sim 4990 \quad \mathrm{~W} /\left(\mathrm{K} \cdot \mathrm{m}^{2}\right)$. Combined with $\kappa_{x}$ and $\kappa_{y}$ measured in section 3.2, we calculated $B i$ numbers under vacuum and atmospheric pressure.

Under $6 \mu$ bar, $B i_{w}<7.2 \times 10^{-3} \ll 0.1$ and $B i_{z}<1.7 \times 10^{-3} \ll 0.1$. This small $B i$ verified the assumption of both $1 \mathrm{D}$ heat conduction and uniform temperature on cross-section. In Fig. 2a, the small value of $B i_{w}$ could also be confirmed by the uniform temperature across the width of both samples.

Under atmospheric pressure, the $B i_{w}<1.9 \times 10^{-2}<0.1$ and $B i_{z}<4.5 \times 10^{-3} \ll 0.1$. These results suggested that even under atmospheric pressure, our modified steady-state method could also be used. And smaller $B i$ numbers under vacuum guaranteed a more uniform temperature distribution in materials, especially for thin materials with a poor out of plane thermal conductivity or anisotropic thermal conductivity.

\section{Conclusions}

With modified steady-state technique and calibrated radiation and convection heat transfer coefficients with standard material, we directly measured the in plane thermal conductivity of CNT sheets. With another steady state method, we calculated the thermal conductivity out of plane. Results illustrated the anisotropic thermal conductivity, with in alignment direction, perpendicular to alignment direction and out of plane direction values as $142.3 \pm 30.1 \mathrm{~W} /(\mathrm{K} \cdot \mathrm{m}), 126.6 \pm 26.2 \mathrm{~W} /(\mathrm{K} \cdot \mathrm{m})$ and $108 \pm 4$ $\mathrm{mW} /(\mathrm{K} \cdot \mathrm{m})$, respectively.

Furthermore, the thermal conductivity of the CNT sheet reinforced polymer composites could also be measured using the same in plane method, as $51.6 \pm 10.7 \mathrm{~W} /(\mathrm{K} \cdot \mathrm{m})$ when the volume fraction of CNT sheet reached $\sim 30 \%$. Thanks to the compatibility of the modified technique to both insulating and highly electrically conductive samples, we showed that the CNT matrix retained its heat conduction pathway in polymer composites.

Our measurements rituals can be adapted to other highly conductive nanomaterials assemblies and their composites, especially materials with small heat capacities, or anisotropic properties, or low electrical conductivity. Thus, it will become an important complement to existing commercial $\kappa$ measurement.

\section{Acknowledgements}

This work was supported by EPSRC project 'Advanced Nanotube Application and Manufacturing (ANAM) Initiative' [grant numbers EP/M015211/1].

The authors thank Tortech Nano Fibers Ltd for offering CNT sheet materials. The authors also thank Dr. Sarah Stevenson, Brian Graves, Dr. Jean de La Verpilliere, Dr. Christian Hoecker and Joe Stallard for their kind support and useful discussion, and Ms Nicola Cavaleri for writing assistance.

Declarations of interest: none.

Supplementary Material: details of convective heat loss coefficient under atmospheric pressure, SEM images and thermal conductivities of composites are available in the online version of this article at https://doi.org/10.17863/CAM.18098

\section{References}

$\$$ When experiment is operated at room temperature and $\mathrm{T} \leq$ $60^{\circ} \mathrm{C}$, the deviation of this simplification is quite small: $\frac{\left(T^{4}-T_{\infty}^{4}\right)-\left[4 T_{\text {mean }}^{3}\left(T-T_{\infty}\right)\right]}{T^{4}-T_{\infty}^{4}}<0.3 \%$

II More details about the CNT sheet could be found at http://tortechnano.com/tortech-nano-fibers

[1] Behabtu N, Young C C, Tsentalovich D E, Kleinerman O, Wang X, Ma A W K, Bengio E A, ter Waarbeek R F, de Jong J J, Hoogerwerf R E, Fairchild S B, Ferguson J B, Maruyama B, Kono J, Talmon Y, Cohen Y, Otto M J and Pasquali M 2013 Strong, Light, Multifunctional Fibers of Carbon Nanotubes with Ultrahigh Conductivity 


\section{Science 339 182-6}

[2] Berber S, Kwon Y K and Tomanek D 2000 Unusually high thermal conductivity of carbon nanotubes Phys. Rev. Lett. 84 4613-6

[3] Bergman T L 2011 Introduction to Heat Transfer: Wiley)

[4] Chen J, Huang X, Zhu Y and Jiang P 2016 Cellulose Nanofiber Supported 3D Interconnected $\mathrm{BN}$ Nanosheets for Epoxy Nanocomposites with Ultrahigh Thermal Management Capability Advanced Functional Materials 1604754-n/a

[5] Churchill S W and Chu H H $\mathrm{S} 1975$ CORRELATING EQUATIONS FOR LAMINAR AND TURBULENT FREE CONVECTION FROM A VERTICAL PLATE Int. J. Heat Mass Transf. 18 1323-9

[6] Fujii M, Zhang X, Xie H Q, Ago H, Takahashi $\mathrm{K}$, Ikuta $\mathrm{T}$, Abe $\mathrm{H}$ and Shimizu T 2005 Measuring the thermal conductivity of a single carbon nanotube Phys. Rev. Lett. 954

[7] Gspann T S, Juckes S M, Niven J F, Johnson M B, Elliott J A, White M A and Windle A H 2017 High thermal conductivities of carbon nanotube films and micro-fibres and their dependence on morphology Carbon 114 160-8

[8] $\mathrm{Hu}$ L, Desai T and Keblinski P 2011 Thermal transport in graphene-based nanocomposite Journal of Applied Physics 1105

[9] $\mathrm{Hu}$ X J, Padilla A A, Xu J, Fisher T S and Goodson K E 2006 3-omega measurements of vertically oriented carbon nanotubes on silicon $J$. Heat Transf.-Trans. ASME 128 1109-13

[10] Ji T X, Feng Y Y, Qin M M and Feng W 2016 Thermal conducting properties of aligned carbon nanotubes and their polymer composites Compos. Pt. A-Appl. Sci. Manuf. 91 351-69

[11] Jiang S H, Liu C H and Fan S S 2014 Efficient Natural-Convective Heat Transfer Properties of Carbon Nanotube Sheets and Their Roles on the Thermal Dissipation ACS Appl. Mater. Interfaces 6 3075-80
[12] Kabo G J, Paulechka E, Blokhin A V, Voitkevich O V, Liavitskaya T and Kabo A G 2016 Thermodynamic Properties and Similarity of Stacked-Cup Multiwall Carbon Nanotubes and Graphite J. Chem. Eng. Data 61 3849-57

[13] Kim P, Shi L, Majumdar A and McEuen P L 2001 Thermal transport measurements of individual multiwalled nanotubes Phys. Rev. Lett. 87

[14] Li Y L, Kinloch I A and Windle A H 2004 Direct spinning of carbon nanotube fibers from chemical vapor deposition synthesis Science 304 276-8

[15] Mahanta N K and Abramson A R 2010 The dualmode heat flow meter technique: A versatile method for characterizing thermal conductivity Int. J. Heat Mass Transf. 53 5581-6

[16] Marconnett A M, Yamamoto N, Panzer M A, Wardle B L and Goodson K E 2011 Thermal Conduction in Aligned Carbon NanotubePolymer Nanocomposites with High Packing Density ACS Nano 5 4818-25

[17] Mayhew E and Prakash V 2014 Thermal conductivity of high performance carbon nanotube yarn-like fibers Journal of Applied Physics 1159

[18] Parker W J, Jenkins R J, Abbott G L and Butler C P 1961 Flash Method of Determining Thermal Diffusivity, Heat Capacity, and Thermal conductivity Journal of Applied Physics 32 1679-\&

[19] Pop E, Varshney V and Roy A K 2012 Thermal properties of graphene: Fundamentals and applications MRS Bulletin 37 1273-81

[20] Raravikar N R, Schadler L S, Vijayaraghavan A, Zhao Y P, Wei B Q and Ajayan P M 2005 Synthesis and characterization of thicknessaligned carbon nanotube-polymer composite films Chem. Mat. 17 974-83

[21] Saidi M and Abardeh R H 2010 Air pressure dependence of natural-convection heat transfer(vol 2) p 1-4

[22] Tan J C, Tsipas S A, Golosnoy I O, Curran J A, 
Paul S and Clyne T W 2006 A steady-state Bisubstrate technique for measurement of the thermal conductivity of ceramic coatings Surf. Coat. Technol. 201 1414-20

[23] Yu A P, Ramesh P, Sun X B, Bekyarova E, Itkis M E and Haddon R C 2008 Enhanced Thermal Conductivity in a Hybrid Graphite Nanoplatelet - Carbon Nanotube Filler for Epoxy Composites Adv. Mater. 20 4740-+

[24] Zhang G, Jiang S H, Yao W and Liu C H 2016 Enhancement of Natural Convection by Carbon Nanotube Films Covered Microchannel-Surface for Passive Electronic Cooling Devices ACS Appl. Mater. Interfaces 8 31202-11

[25] Zhang X, Song L, Cai L, Tian X Z, Zhang Q, Qi X Y, Zhou W B, Zhang N, Yang F, Fan Q X, Wang Y C, Liu H P, Bai X D, Zhou W Y and Xie S S 2015 Optical visualization and polarized light absorption of the single-wall carbon nanotube to verify intrinsic thermal applications Light-Sci. Appl. 48

[26] Zhang X, Yang F, Zhao D, Cai L, Luan P, Zhang Q, Zhou W, Zhang N, Fan Q, Wang Y, Liu H, Zhou W and Xie S 2014 Temperature dependent Raman spectra of isolated suspended singlewalled carbon nanotubes Nanoscale 6 3949-53

[27] Zhou W B, Fan Q X, Zhang Q, Li K W, Cai L, Gu X G, Yang F, Zhang N, Xiao Z J, Chen H L, Xiao S Q, Wang Y C, Liu H P, Zhou W Y and Xie S S 2016 Ultrahigh-Power-Factor Carbon Nanotubes and an Ingenious Strategy for Thermoelectric Performance Evaluation Small 12 3407-+ 


\section{Supplementary Material}

\section{High-fidelity Characterization on Anisotropic Thermal Conductivity of Carbon Nanotube Sheets and on Their Effects of Thermal Enhancement of Nanocomposites}

Xiao Zhang ${ }^{\mathrm{a}}(\bowtie)$, Wei Tan ${ }^{\mathrm{b}}$, Fiona Smail ${ }^{\mathrm{a}}$, Michael De Volder ${ }^{\mathrm{c}}$, Norman Fleck $^{\mathrm{b}}$, Adam Boies $^{\mathrm{a}}(\bowtie)$

${ }^{a}$ Division of Energy, Department of Engineering, University of Cambridge, Cambridge, CB2 1PZ, UK

${ }^{\mathrm{b}}$ Division of Mechanics, Department of Engineering, University of Cambridge, Cambridge, CB2 1PZ, UK

${ }^{\mathrm{c}}$ Institute for Manufacturing, Department of Engineering, University of Cambridge, Cambridge, CB3 OFS, UK 


\section{Material and Method}

A-1. Calculation of Rayleigh number, average Nusselt number and average convective coefficient.

The Rayleigh number $(R a)$ was calculated with the following expression:

$$
R a=\frac{g \beta\left(T-T_{g}\right) w^{3}}{v \alpha}
$$

here, standard gravity $g=9.8 \mathrm{~m} / \mathrm{s}^{2} ; \beta$ represents the expansion coefficient, for an ideal gas, $\beta=\frac{1}{T}$; T and $T_{g}$ represent the temperature at sample surface and free gas out of boundary layer; $w$ represents the width of sample; $v$ and $\alpha$ represent the kinematic viscosity and heat diffusivity of gas.

Based on our experiment results and the thermophysical properties data in Table S1 by temperature interpolation., the $R a$ was around 30-400, which is far below the critical $R a=10^{9}$ to develop a turbulent boundary layer.

Thus, the average Nusselt number $(\overline{N u})$ was calculated based on the following correlation[1]:

$$
\bar{N} \bar{u}=\frac{\overline{h_{c}} w}{\kappa}=\frac{4}{3}\left(\frac{G r}{4}\right)^{\frac{1}{4}} g(\operatorname{Pr})
$$

here, $\overline{h_{c}}$ and $\kappa$ represent the average convective coefficient and thermal conductivity of gas, Grashof number $G \mathrm{r}=$ $\frac{\mathrm{g} \beta\left(\mathrm{T}-\mathrm{T}_{\mathrm{g}}\right) \mathrm{w}^{3}}{\mathrm{v}^{2}}$, Prandtl number $\operatorname{Pr}=\frac{v}{\alpha}$ and $g(\operatorname{Pr})$ is an empirical correlation as follows:

$$
g(\operatorname{Pr})=\frac{0.75 \operatorname{Pr}^{1 / 2}}{\left(0.609+1.221 \operatorname{Pr}^{\frac{1}{2}}+1.238 P r\right)^{1 / 4}}
$$

Furthermore, a better fitting result for air could be obtained by using the following empirical correlation[2]:

$$
\overline{N u}=0.680+\frac{0.670 R a^{1 / 4}}{\left[1+(0.492 / P r)^{9 / 16}\right]^{4 / 9}}
$$

\begin{tabular}{|c|c|c|c|c|c|c|c|c|}
\hline Gas & $\begin{array}{c}\text { T of Gas } \\
{[\mathrm{K}]}\end{array}$ & $\begin{array}{c}\text { Density }(\rho) \\
{\left[\mathrm{kg} / \mathrm{m}^{3}\right]}\end{array}$ & $\begin{array}{c}c_{p} \\
{[\mathrm{~kJ} / \mathrm{kg} / \mathrm{K}]}\end{array}$ & $\begin{array}{c}\text { Viscosity }(\mu) \\
{[\mathrm{Pa} \cdot \mathrm{s}]}\end{array}$ & $\begin{array}{c}v \\
{\left[\mathrm{~m}^{2} / \mathrm{s}\right]}\end{array}$ & $\begin{array}{c}\alpha \\
{\left[\mathrm{m}^{2} / \mathrm{s}\right]}\end{array}$ & $\begin{array}{c}\kappa \\
{[\mathrm{W} / \mathrm{K} / \mathrm{m}]}\end{array}$ & $\begin{array}{c}\text { Calculated } \overline{h_{c}} \\
{\left[\mathrm{~W} / \mathrm{K} / \mathrm{m}^{2}\right]}\end{array}$ \\
\hline \multirow{3}{*}{ Air[1] } & 250 & 1.39 & 1.006 & $1.60 \mathrm{E}-05$ & $1.14 \mathrm{E}-05$ & $1.59 \mathrm{E}-05$ & 2.23E-02 & \multirow{3}{*}{$\begin{array}{l}\text { 4.6-8.2 (Eq.S2) } \\
7.1-10.8 \text { (Eq.S4) }\end{array}$} \\
\hline & 300 & 1.16 & 1.007 & $1.85 \mathrm{E}-05$ & $1.59 \mathrm{E}-05$ & $2.25 \mathrm{E}-05$ & 2.63E-02 & \\
\hline & 350 & 1.00 & 1.009 & $2.08 \mathrm{E}-05$ & $2.09 \mathrm{E}-05$ & 2.99E-05 & $3.00 \mathrm{E}-02$ & \\
\hline \multirow{3}{*}{$\mathrm{He}[1]$} & 260 & 0.19 & 5.193 & $1.80 \mathrm{E}-05$ & $9.60 \mathrm{E}-05$ & $1.41 \mathrm{E}-04$ & $1.37 \mathrm{E}-01$ & \multirow{3}{*}{$\begin{array}{l}9.1-16.9(\text { Eq.S2) } \\
23.3-31.5 \text { (Eq.S4) }\end{array}$} \\
\hline & 300 & 0.16 & 5.193 & $1.99 \mathrm{E}-05$ & $1.22 \mathrm{E}-04$ & $1.80 \mathrm{E}-04$ & $1.52 \mathrm{E}-01$ & \\
\hline & 400 & 0.12 & 5.193 & $2.43 \mathrm{E}-05$ & $1.99 \mathrm{E}-04$ & $2.95 \mathrm{E}-04$ & $1.87 \mathrm{E}-01$ & \\
\hline \multirow{4}{*}{$\operatorname{Ar}[3]$} & 280 & 1.72 & 0.521 & $2.16 \mathrm{E}-05$ & $1.26 \mathrm{E}-05$ & $1.89 \mathrm{E}-05$ & $1.69 \mathrm{E}-02$ & \multirow{4}{*}{$\begin{array}{l}2.9-5.8 \text { (Eq.S2) } \\
4.5-7.5 \text { (Eq.S4) }\end{array}$} \\
\hline & 300 & 1.60 & 0.521 & 2.29E-05 & $1.43 \mathrm{E}-05$ & $2.15 \mathrm{E}-05$ & $1.79 \mathrm{E}-02$ & \\
\hline & 320 & 1.52 & 0.521 & $2.42 \mathrm{E}-05$ & $1.59 \mathrm{E}-05$ & 2.39E-05 & $1.89 \mathrm{E}-02$ & \\
\hline & 340 & 1.40 & 0.521 & $2.54 \mathrm{E}-05$ & $1.82 \mathrm{E}-05$ & $2.73 \mathrm{E}-05$ & 1.99E-02 & \\
\hline
\end{tabular}

Table S1. Thermophysical Properties of Gases at Atmospheric Pressure 


\section{A-2. SEM image of CNT mat and CNT mat reinforced composite.}

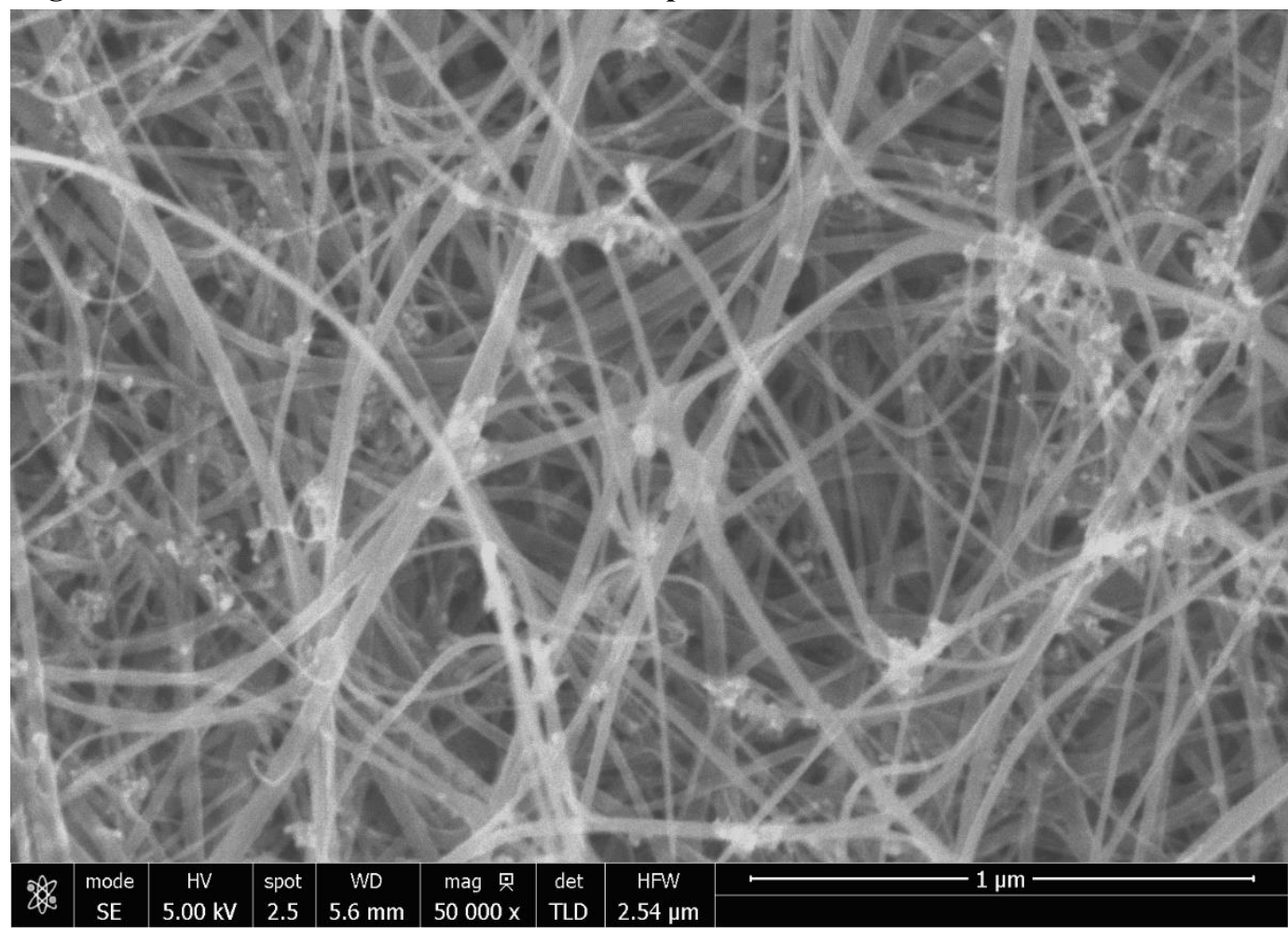

Figure S-1 SEM image of pure CNT mat for directly measurement and thermal enhancement filler in CNT reinforced polymer composites.

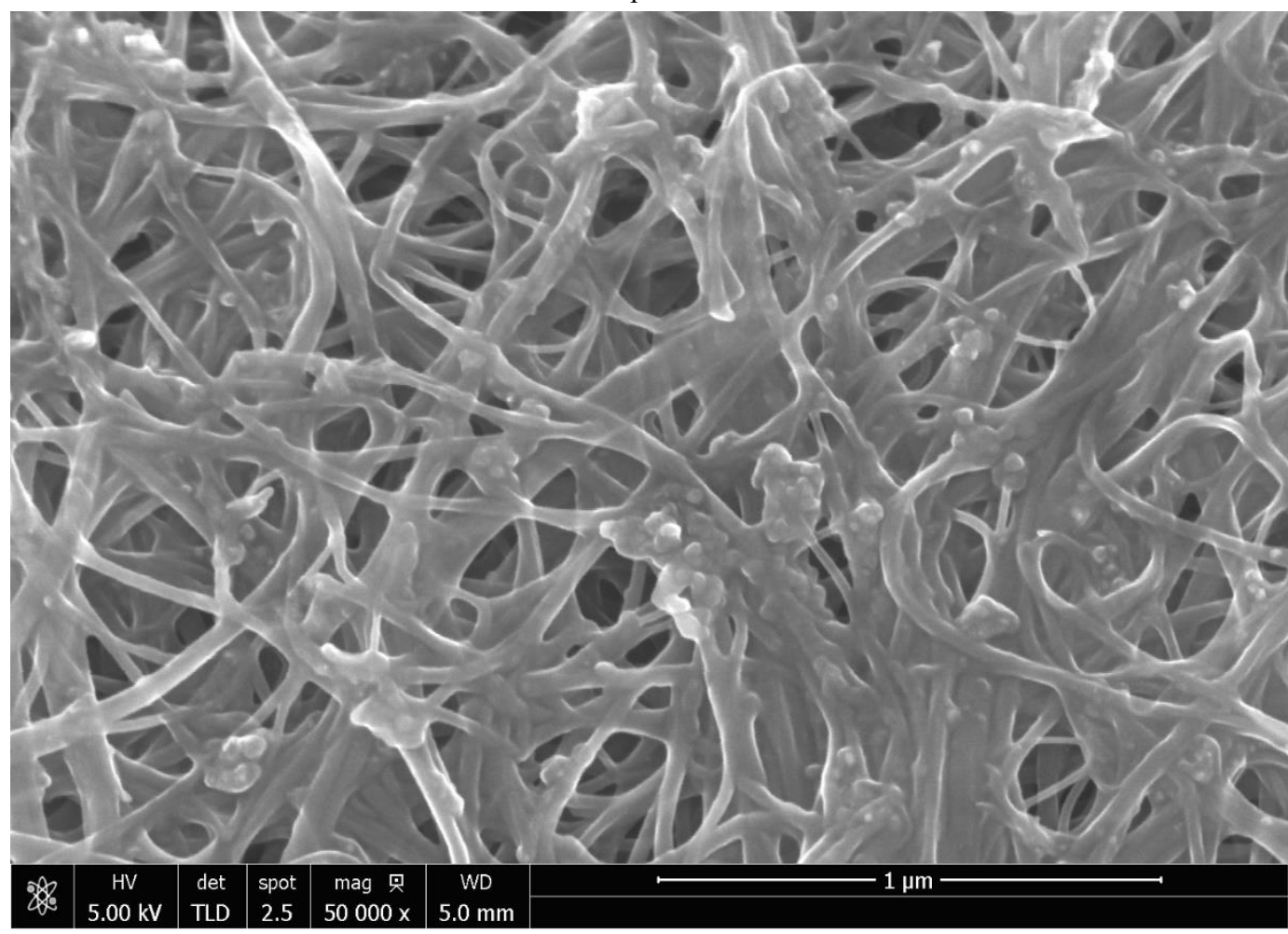

Figure S-2 SEM image of CNT reinforced polymer composites with 24 vol\% of CNTs. 

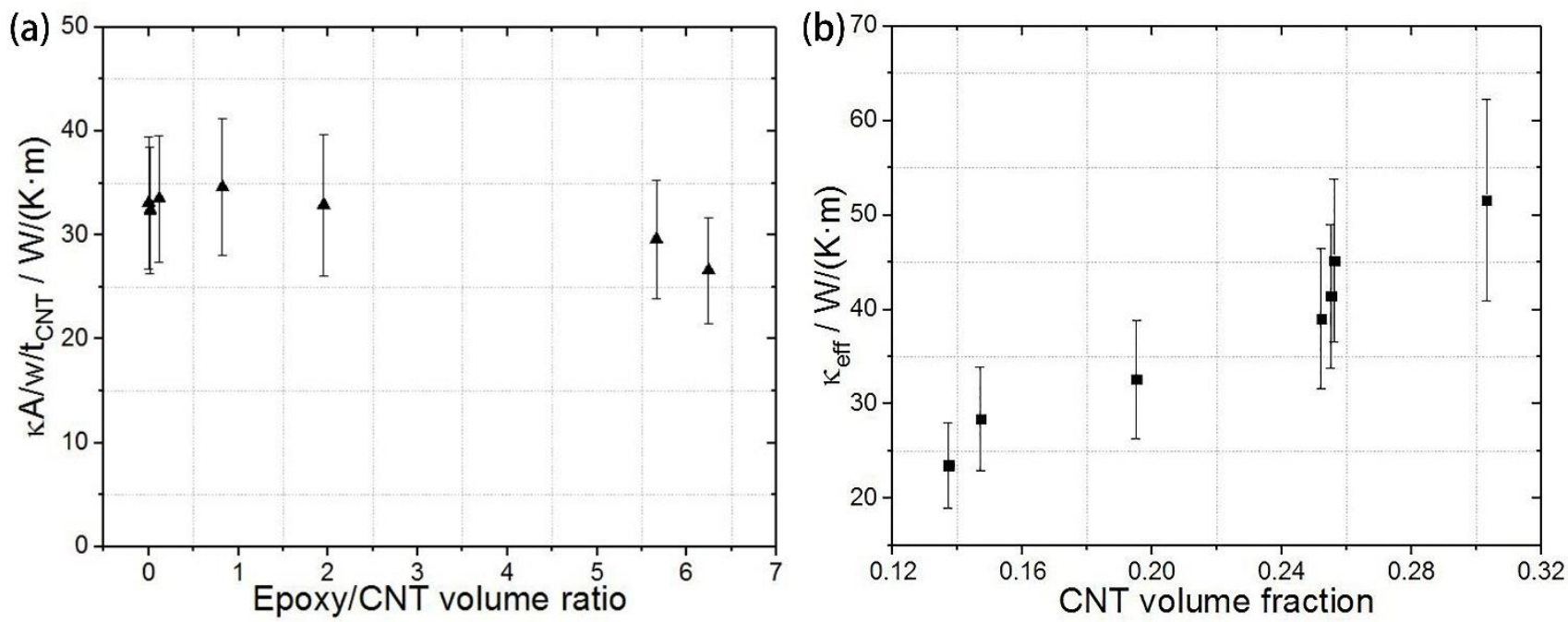

Figure S-3 (a) In CNT mat reinforced composite, with epoxy increasing in composite, $\kappa A$ normalized by CNT strips' width ( $w$ ) and thickness $\left(t_{C N T}\right)$ preserved its value in pure CNT mat; (b) with CNT volume fraction increase in composite, $\kappa_{c p}^{e f f}$ kept increasing.

\section{References}

[1] Bergman T L 2011 Introduction to Heat Transfer: Wiley

[2] Churchill S W and Chu H H S 1975 CORRELATING EQUATIONS FOR LAMINAR AND TURBULENT FREE CONVECTION FROM A VERTICAL PLATE Int. J. Heat Mass Transf. 18 1323-9

[3] Haynes W M 2014 CRC Handbook of Chemistry and Physics, 95th Edition: CRC Press 